\title{
Five new species of Oedogonium Link (Chlorophyta), a freshwater filamentous algae from Nepal
}

\author{
Shiva Kumar Rai \\ Department of Botany, P.G. Campus, T.U., Biratnagar, Nepal \\ E-mail: sk.khaling@gmail.com
}

\begin{abstract}
The present paper deals with six species of genus Oedogonium Link, among which five species are being recorded for the first time from Nepal. They are Oedogonium leave Wittrock, O. peipingense Jao, $O$. pisanum Wittrock, O. plagiostomum Wittrock and O. undulatum (Bréb.) A. Braun in De Bary.
\end{abstract}

Key words: Oedogonium, Chlorophyceae, filamentous algae, Nepal

\section{Introduction}

Filamentous green algae of Nepal have not been studied adequately so far. Rai and Misra (2007) have reported 11 species of Spirogyra Link and 1 species of Sirogonium Kützing from east Nepal, all were new to Nepal. Zygnema pectinatum Vauch. Ag. is the single species reported from the country from the ponds at Godawari and Thimi (Shrestha \& Manandhar, 1983; Haga, 1988). There are four species of Ulothrix reported from Nepal viz., U. subconstricta West, U. tenuissima Kutz. and U. variabilis Kutz. from Gaindakot, Tikauli and Narayanghat (Das \& Verma, 1996) and U. zonata Weber et Mohr Kutz. from Patan and Thimi (Shrestha \& Manandhar, 1983). Other filamentous chlorophyceae reported from Nepal belong to 6 taxa of Trentepohlia viz., T. arborum, T. aurea, T. aurea var. polycarpa, T. aurea var. tenuior, T. effuse var. subtropica and T. monilia growing on bark of trees in Janakpur and Kathmandu (Dwivedi, 1985; Akiyama, 1989). Similarly, a total 11 taxa of Stigeoclonium have been reported as $S$. dejectum and $S$. echinalum from Khumbu Himal (Kusel-fetzmann, 1969), S. flagelliferum from Kirtipur (Shrestha \& Manandhar, 1983), S. amoenum and $S$. nanum from Janakpur (Dwivedi, 1985), S. elongatum from Rara lake (Watanabe, 1995), S. stagnale from Tandi (Das \& Verma, 1996) and S. farctum, S. fasciculare, S. flagelliferum and $S$. pachydermum from Koshi Tappu (Kargupta \& Jha, 1997). Dwivedi (1985) has also reported 6 taxa of chaetophorales from Janakpur, all new for the country. Haga (1988) of Hokkaido University has reported 9 species of filamentous chlorophyceae from Kathmandu among which Geminella minor, Klebsormidium pseudomirabile, Uronema confervicolum, U. gigas and Gongrosira papuasia were new for Nepal.

Literature reveal that only five species of Oedogonium are reported from Nepal hitherto (Prasad, 2011). They are O. cardiacum (Hass.) Wittr. reported from Kathmandu (Shrestha \& Manandhar, 1983); O. rothii (Le Cl) Pringsh. and O. tapeinosportum Wittr. from Rara lake (Watanabe, 1995); and O. pseudaureum Jao and O. nanum Wittr. from Mahendranagar (Habib, 1997). Thus an attempt was made to study the Oedogonium algae from east Nepal. This report may help to add the filamentous green algae to the algal database of Nepal. 


\section{Materials and Methods}

Algal samples were collected from different localities of eastern Nepal among which the samples collected from Koshi Barage reservoir, Titrigachi pond, Sawane-Thingabari rice field, roadside ditches at Tarahara and Rauta pokhari bear the specimens of Oedogonium (Fig. 1). Algae were picked up simply by hand from shallow edges of the ponds or occasionally by plankton net and then preserved in $4 \%$ formaldehyde solution. Preliminary screening of Oedogonium species from the samples was made in the Algae Research Lab, Department of Botany, P.G. Campus. Microphotography and measurement of specimens were carried out with the help of Olympus binocular microscope model $\mathrm{CH} 20 \mathrm{i}$ and Canon digital camera. Identification was made with the help of relevant literature following Prescott (1951), Tiffany and Britton (1952), Gonzalves (1982) etc.

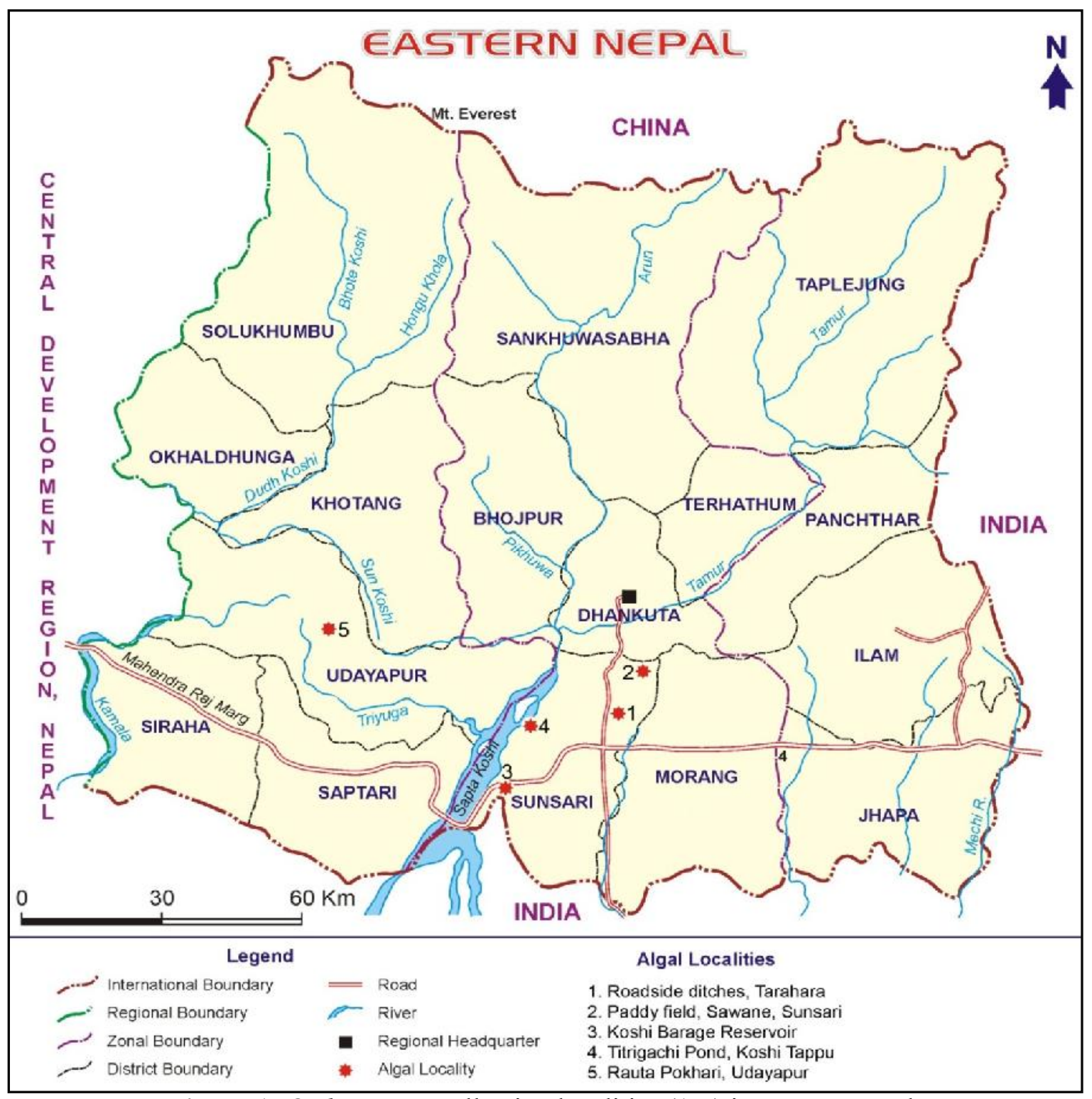

Figure 1. Oedogonium collection localities (1-5) in eastern Nepal. 


\section{Results}

\section{Systematic position}

Phylum: Chlorophyta

Subphylum: Tetraphytina

Class: Chlorophyceae

Order: Oedogoniales

Family: Oedogoniaceae

Genus: Oedogonium Link 1820

\section{Genus character}

Filament single, unbranched and attached or free-floating; vegetative cell uninucleate, cylindrical, or sometimes capitellate, nodulose or undulate and anterior end with caps or ring-like scars formed by cell division; basal cell having holdfast; terminal cell obtuse, apiculate or hyaline; chloroplast parietal, reticulate with numerous pyrenoids.

Reproduction by multiflagellate zoospores; oogonia and eggs, antheridia and multiflagellate sperms, oospores; dwarf males, androsporangia, androspores.

\section{Key to the species}

1. Dwarf male plants present, attached on or near oogonia; walls of vegetative cells undulate; vegetative cells $66 \mu \mathrm{m}$ long, $18.7 \mu \mathrm{m}$ broad.

1. Dwarf male plants absent. 2

2. Oogonia opening by a pore; wall of oospores smooth. 3

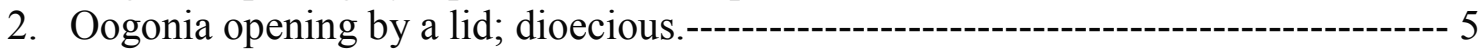

3. Monoecious; oogonia depressed-globose, $24-29.5 \mu \mathrm{m}$ long, $24.5-30 \mu \mathrm{m}$ broad.--- $O$. laeve

3. Dioecious; oospores globose, subglobose or cylindric globose.------------------------- 4

4. Female vegetative cells $40-60 \mu \mathrm{m}$ long, $20 \mu \mathrm{m}$ broad; oogonia $45 \mu \mathrm{m}$ long, $41 \mu \mathrm{m}$ in diameter. O. plagiostomum

4. Female vegetative cells $110-150 \mu \mathrm{m}$ long, 30-32 $\mu \mathrm{m}$ broad; oogonia $80 \mu \mathrm{m}$ long, 66 $\mu \mathrm{m}$ in diameter. O. peipingense

5. Operculum median; oogonia depressed-globose or subpyriform-globose, 19.5-21.5 $\mu \mathrm{m}$ long, $19 \mu \mathrm{m}$ in diameter; oospores not filling the oogonia. O. tapeinosporum

5. Operculum superior; oogonia ovoid to ellipsoid-ovoid, $40 \mu \mathrm{m}$ long, $36 \mu \mathrm{m}$ in diameter.-O. pisanum

\section{Taxonomic description}

\section{Oedogonium laeve Wittrock (Pls. 1-2)}

Prescott, G.W. 1951, p. 179; Tiffany, L.H. and Britton, M.E. 1952, p. 64, pl. 17, fig. 130.

Taxonomic characters: Monoecious, macrandrous; vegetative cell cylindrical, 25-29 $\mu \mathrm{m}$ long, 9-10 $\mu \mathrm{m}$ broad; oogonium solitary, depressed globose, pore median, 24-29.5 $\mu \mathrm{m}$ long, 24.5-30 $\mu \mathrm{m}$ broad; oospore depressed globose, filling oogonia, wall smooth, 23-26 $\mu \mathrm{m}$ long, 30-35 $\mu \mathrm{m}$ broad; antheridia not seen.

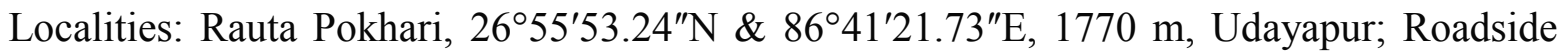
ditches at Tarahara, $26^{\circ} 42^{\prime} 25.36^{\prime \prime} \mathrm{N} \& 86^{\circ} 16^{\prime} 36.47^{\prime \prime} \mathrm{E}, 142.98 \mathrm{~m}$, Sunsari. 
Collection numbers and dates: EN 300 (03-11-2004); EN 302 (10-09-2004).

Distribution in Nepal: New record for Nepal.

2. Oedogonium peipingense Jao (P1. 3)

Gonzalves, E.A. 1982, p. 334, fig. 9.227; Misra et al. 2002, p. 108, pl. 1, figs. 11, 14.

Taxonomic characters: Female vegetative cell 110-150 $\mu \mathrm{m}$ long, 30-32 $\mu \mathrm{m}$ broad; oogonium $80 \mu \mathrm{m}$ long, $66 \mu \mathrm{m}$ in diameter.

Locality: Roadside ditches at Tarahara, 26²'25.36"N \& 86 $16^{\circ} 36.47^{\prime \prime} \mathrm{E}, 142.98 \mathrm{~m}$, Sunsari.

Collection number and date: EN 302 (10-09-2004).

Distribution in Nepal: New record for Nepal.

World distribution: India (Misra et al., 2002).

3. Oedogonium pisanum Wittrock (P1. 4)

Prescott, G.W. 1951, p. 186, pl. 36, figs. 8-9; Tiffany, L.H. and Britton, M.E. 1952, p. 80, pl. 22, figs. 196-197.

Taxonomic characters: Dioecious, macrandrous; vegetative cell cylindrical, 40-55 $\mu \mathrm{m}$ long, 13-16 $\mu \mathrm{m}$ broad; oogonium solitary, ellipsoid to ovoid, operculate, division superior, $40 \mu \mathrm{m}$ long, $36 \mu \mathrm{m}$ broad; oospore globose, nearly filling oogonium, wall smooth, 18-37 $\mu \mathrm{m}$ long, 18-25 $\mu \mathrm{m}$ broad; antheridium 5-8 $\mu \mathrm{m}$ long, 6-8 $\mu \mathrm{m}$ broad.

Locality: Paddy fields at Sawane-Thingabari, $26^{\circ} 47^{\prime} 40.50^{\prime \prime} \mathrm{N} \& 87^{\circ} 19^{\prime} 26.94^{\prime \prime} \mathrm{E}, 495 \mathrm{~m}$, Panchakanya, Sunsari.

Collection number and date: EN 24 (30-08-2002).

Distribution in Nepal: New record for Nepal.

World distribution: Britain (Huxley \& Pentecost, 2002), Portugal (Cambra Sánchez et.al., 1998), Spain (Alvárez Cobelas \& Gallardo 1986), Victoria (Day et al., 1995).

\section{Oedogonium plagiostomum Wittrock (P1. 5)}

Prescott, G.W. 1951, p. 173, pl. 32, figs. 3-4; Tiffany, L.H. and Britton, M.E. 1952, p. 70, pl. 18, figs. 142-143.

Taxonomic characters: Dioecious, macrandrous; vegetative cell cylindrical, rather stout, 40$60 \mu \mathrm{m}$ long, $20 \mu \mathrm{m}$ broad; oogonium solitary, obovoid-globose, pore superior, $45 \mu \mathrm{m}$ long, $41 \mu \mathrm{m}$ broad; oospore globose, wall thick and smooth, $40 \mu \mathrm{m}$ broad; antheridium $8-12 \mu \mathrm{m}$ long, 20-24 $\mu \mathrm{m}$ broad.

Locality: Roadside ditches at Tarahara, 26²'25.36"N \& 86 $16^{\circ} 36.47^{\prime \prime} \mathrm{E}, 142.98 \mathrm{~m}$, Sunsari. Collection number and date: EN 302 (10-09-2004).

Distribution in Nepal: New record for Nepal.

World distribution: India (Anonym, 2012), Queensland (Bostock \& Holland, 2010). 

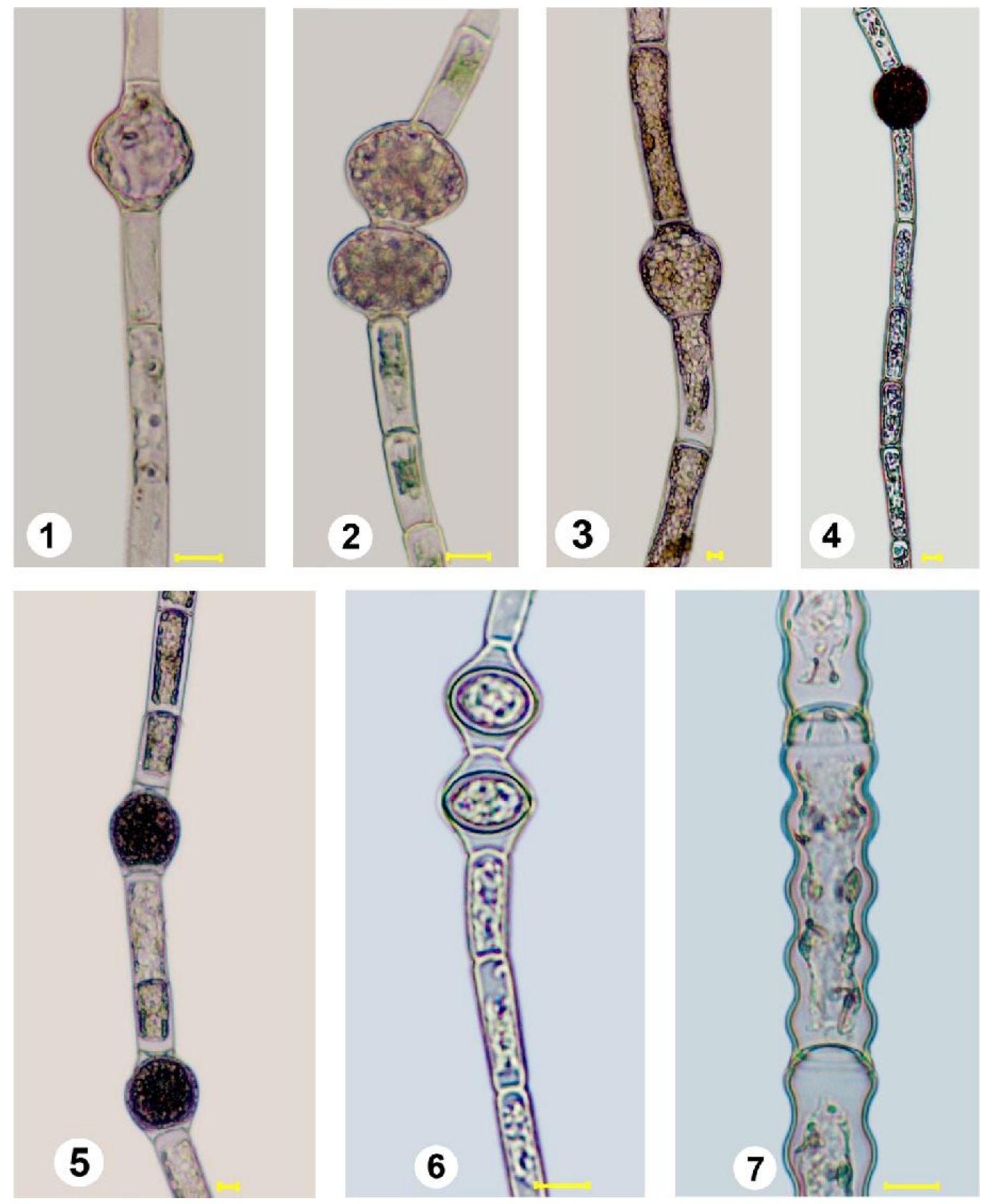

Plates 1-2. Oedogonium laeve Wittrock, Plate 3. O. peipingense Jao, Plate 4. O. pisanum Wittrock, Plate 5. O. plagiostomum Wittrock, Plate 6. O. tapeinosporum Wittrock, Plate 7. O. undulatum (Bréb.) A. Braun in De Bary. (Scale bar in each plate $=10 \mu \mathrm{m}$ ) 


\section{Oedogonium tapeinosporum Wittrock (P1. 6)}

Prescott, G.W. 1951, p. 188, pl. 38, figs. 11-12; Watanabe, M. 1995, p. 10, fig. 27.

Taxonomic characters: Dioecious, macrandrous; vegetative cell cylindrical, 22-27 $\mu \mathrm{m}$ long, 5-8 $\mu \mathrm{m}$ broad, basal cell hemispherical; oogonium solitary, globose to subelliptical, operculate, division median, 19.5-21.5 $\mu \mathrm{m}$ long, $19 \mu \mathrm{m}$ broad; oospore globose, not filling oogonium, wall smooth, $17-17.5 \mu \mathrm{m}$ long, 12.5-13 $\mu \mathrm{m}$ broad; antheridium not seen.

Locality: Titrigachi pond, $26^{\circ} 37^{\prime} 04.16^{\prime \prime} \mathrm{N} \& 8^{\circ} 01^{\prime} 27.99^{\prime \prime} \mathrm{E}, 216$ m, Koshi Tappu, Kusaha, Sunsari.

Collection number and date: EN 101 (21-12-2002).

Distribution in Nepal: A small pond south of Rara lake, 3,030 m, Kaski (Watanabe, 1995). New for eastern Nepal.

World distribution: Britain (Huxley \& Pentecost, 2002), Portugal (Cambra Sánchez et.al., 1998), Queensland (Bostock \& Holland, 2010).

6. Oedogonium undulatum (Bréb.) A. Braun in De Bary (P1. 7)

Prescott, G.W. 1951, p. 209, pl. 40, figs. 3-5; Tiffany, L.H. and Britton, M.E. 1952, p. 84, pl. 24, fig. 229.

Taxonomic characters: Dioecious, nannandrous, gynandrosporous or idioandrosporous; vegetative cell capitellate, 4-5 undulation per cell, $66 \mu \mathrm{m}$ long, $18.7 \mu \mathrm{m}$ broad, constrictions $14 \mu \mathrm{m}$ wide, basal cell elongate and not undulate; oogonium solitary or two, subglobose or ellipsoid-globose, operculate, division inferior, wide; oospore globose, filling the oogonia, wall smooth, $48 \mu \mathrm{m}$ broad; antheridium not seen.

Locality: Water reservoir south of highway adjacent to the boarder of India, between Koshi Barrage and Bhantabari, 26³1'14.60"N \& 86 57'08.44"E, 162 m, Sunsari.

Collection number and date: EN 128 (29-03-2003).

Distribution in Nepal: New record for Nepal.

World distribution: Britain (Huxley \& Pentecost, 2002), China (Hu \& Wei, 2006), New South Wales, Northern Territory, Pakistan (Munir et al., 2007), Queensland, Romania (Caraus, 2012), Spain (Cambra Sánchez et.al., 1998), Victoria (Day et al., 1995).

\section{References}

Akiyama, M. 1989. Some Trentepohlia species from Kathmandu, Nepal Himalaya. Bull. Natn. Sci. Mus., Ser. B, Tokyo. 15(3): 81-90.

Alvárez Cobelas, M. \& T. Gallardo. 1986. Catálogo de las algas continentales españolas. IV. Chlorophyceae Wille in Warming 1884. Prasinophyceae T. Christensen ex Silva 1980. Acta Bot. Malacitana 11: 17-38.

Anonym. 2012. Punjab ENVIS Centre, set up at Punjab State Council for Science \& Technology (PSCST), Chandigarh, India. List of Algae http://www.punenvis.nic.in/DATA BASE_Tables/Biodiversity_Database/db_bd01.htm. browse on 24 September, 2012. 
Bostock, P.D. \& A.E. Holland. 2010. Census of the Queensland Flora. Brisbane: Queensland Herbarium Biodiversity and Ecosystem Sciences, Department of Environment and Resource Management. pp. 1-320.

Cambra Sánchez, J., M. Álvarez Cobelas \& M. Aboal Sanjurjo. 1998. Lista florística y bibliográfica de los clorófitos (Chlorophyta) de la Península Ibérica, Islas Baleares e Islas Canarias. Burgos: Asociación Española de Limnología. pp. 1-614.

Caraus, I. 2012. Algae of Romania. A distributional checklist of actual algae. Version 2.3 third revision. . Bacau: Univ. Bacau.

Das, S.N. \& B.N. Verma. 1996. Algal flroa of Chitwan and Nawalparasi districts of Nepal. Phykos 35(1-2): 119-127.

Day, S.A., R.P. Wickham, T.J. Entwisle \& P.A. Tyler. 1995. Bibliographic check-list of nonmarine algae in Australia. Flora of Australia Supplementary Series 4: 276.

Dwivedi, N.P. 1985. Some new records of Chaetophorales from Nepal. Mendel 1-2(2):120-123.

Gonzalves, E.A. 1982. Oedogoniales, I.C.A.R. monograph on algae, New Delhi. 757p.

Habib, I. 1997. Algal flora from Mahendranagar, Nepal. J. Econ. \& Taxon. Bot., India. 21(1): 19-26.

Haga, M. 1988. A brief note on filamentous Chlorophyceae from Kathmandu. In: Cryptogams of the Himalayas, Vol. 1, The Kathmandu Valley (Eds. M. Watanabe \& S.B. Malla). National Science Museum, Tsukuba, Japan. pp. 47-55.

Hu, H. \& Y. Wei. 2006. The freshwater algae of China. Systematics, taxonomy and ecology. China. pp. 1-1023. www.sciencep.com. Browse on $24^{\text {th }}$ Sept., 2012.

Huxley, R. \& A. Pentecost. 2002. Order Oedogoniales. In: The Freshwater Algal Flora of the British Isles. An identification guide to freshwater and terrestrial algae (Eds. D.M. John, B.A. Whitton \& A.J. Brook). Cambridge: Cambridge University Press. pp. 409-432.

Kargupta, A.N. \& S. Jha. 1997. The genus Stigeoclonium from North Bihar and bordering areas of Nepal. Phykos 36(1-2): 37-42.

Kusel-fetzmann, E. 1969. Einige Algen aus Nepal. Khumbu Himal (Berlin) 1(6): 37-56.

Misra, P.K., A.K. Srivastava, R.K. Mehrotra and S.K. Singh. 2002. Genus Oedogonium Link from Northeastern Uttar Pradesh. Geophytology 30(1-2): 103-109.

Munir, M.A., A. Zarina, Masud-ul-Hasan \& M. Shameel. 2007. Occurrence of the genus Oedogonium (Zygnemophyceae Shameel) in a freshwater pond of Lahore, Pakistan. International Journal of Phycology \& Phycochemistry 3(1): 71-74.

Prasad, V. 2011. Modern check-list of algae of Nepal. S. Devi, Manipal House, Vishwa, Birgunj, Nepal. 84p.

Prescott, G.W. 1951. Algae of the western great lakes area. WM.C. Brown Publishers, Dubuque, Iowa. 977p.

Rai, S.K. \& P.K. Misra. 2007. Spirogyra Link and Sirogonium Kutzing species: New to algal flora of Nepal. Ecoprint 14: 89-96.

Shrestha, B. \& J.D. Manandhar. 1983. Contribution to the algal flora of Kathmandu valley. $J$. Inst. Sci. Techn., Nepal. 6:1-6.

Tiffany, L.H. \& M.E. Britton. 1952. The algae of Illinois. Hafner Publishing Co., New York. $407 \mathrm{p}$.

Watanabe, M. 1995. Algae from lake Rara and its vicinities, Nepal Himalayas. In: Cryptogams of the Himalayas, Vol. 3, Nepal and Pakistan (Eds. M. Watanabe and H. Hagiwara). National Science Museum, Tsukuba, Japan. pp. 1-17. 